\title{
World Journal of Prevention and control strategies for emergency, limited-term, and elective operations in pediatric surgery during the epidemic period of COVID-19
}

Daxing Tang, ${ }^{1,2}$ Jinfa Tou, ${ }^{1,2}$ Jinhu Wang, ${ }^{1,2}$ Qingjiang Chen, ${ }^{1,2}$ Wei Wang, ${ }^{2,3}$ Jinjin Huang, ${ }^{2,4}$ Hangyan Zhao, ${ }^{2,4}$ Jia Wei, ${ }^{1,2}$ Zheming Xu (D) , ${ }^{1,2}$ Dongyan Zhao, ${ }^{1,2}$ Junfen Fu, ${ }^{2,5}$ Qiang Shu ${ }^{1,2}$

To cite: Tang D, Tou J, Wang J, et al. Prevention and control strategies for emergency, limited-term, and elective operations in pediatric surgery during the epidemic period of COVID-19. World Jnl Ped Surgery 2020;3:e000122. doi:10.1136/ wjps-2020-000122

Received 3 March 2020 Revised 18 March 2020 Accepted 19 March 2020
Check for updates

(c) Author(s) (or their employer(s)) 2020. Re-use permitted under CC BY-NC. No commercial re-use. See rights and permissions. Published by BMJ.

'Department of Pediatric Surgery, Children's Hospital, Zhejiang University School of Medicine, Hangzhou, China ${ }^{2}$ National Clinical Research Center for Child Health, Hangzhou, China ${ }^{3}$ Department of Cardiology, Children's Hospital, Zhejiang University School of Medicine, Hangzhou, China

${ }^{4}$ Anesthesiology and Operation Center, Children's Hospital, Zhejiang University School of Medicine, Hangzhou, China ${ }^{5}$ Department of Endocrinology, Children's Hospital, Zhejiang University School of Medicine, Hangzhou, China

Correspondence to Dr Qiang Shu; shuqiang@zju. edu.cn

\section{ABSTRACT}

The outbreak of coronavirus disease 2019 (COVID-19) caused by severe acute respiratory syndrome coronavirus 2 (SARS-CoV-2) has spread to more than 100 countries. Children approved to be susceptible to SARS-CoV-2 infection. Preventing and controlling the epidemic while ensuring orderly flows of pediatric surgery clinical work has proven to be a big challenge for both patients and clinicians during the epidemic. Based on the transmission characteristics of SARS-CoV-2 and the requirements for prevention and control of COVID-19, the authors proposed some concrete measures and practical strategies of managing emergency, limited-term, and elective pediatric surgeries during the epidemic period.

The epidemic of a novel coronavirus infectionhas spread to more than 100 countries. On January 31, 2020, the World Health Organization (WHO) announced that the outbreak of the new coronavirus constitutes a "public health emergency of international concern (PHEIC)". On February 11, 2020, the International Virus Classification Commission named this virus severe acute respiratory syndrome coronavirus 2 (SARS-CoV-2). ${ }^{1}$ On the same day, WHO named the disease caused by SARS-CoV-2 infection as coronavirus disease 19 (COVID-19). ${ }^{2}$ On January 20, 2020, the National Health Commission of China categorized COVID-19 as a Class $\mathrm{B}$ infectious disease but administered it as a Class A infectious disease. ${ }^{3}$ Many countries and regions were involved in this epidemic and the number of infected cases continues to increase. Some countries and regions implemented very stringent prevention and control measures. Preventing and controlling the epidemic while ensuring the orderly flows of pediatric surgery clinical work has proven to be a big challenge for both patients and clinicians during the epidemic period. Several publications that introduced the prevention strategies for surgical operations during the COVID-19 epidemic have been launched. ${ }^{4-6}$ Because children are susceptible to SARS-CoV-2, ${ }^{7-9}$ several diagnostic guidelines or consensuses for children were published. ${ }^{9-16}$ However, thus far, no publications providing systemic suggestions or strategies for prevention of COVID-19 in the field of pediatric surgery have been identified.

Based on the "Technical Guidelines for the Prevention and Control of New Coronavirus Infection in Medical Institutions (First Edition),"17 "Diagnosis and Treatment Plan on the New Coronavirus inflicted pneumonia (Sixth trial edition, revised)" ${ }^{2}$ (both released by the National Health Commission of China), "Recommendations for the Prevention and Control of General Surgery in the Background of New Coronavirus Outbreak, ${ }^{, 6}$ and other relevant latest reports, we propose the following control measures and practical strategies for pediatric surgery practice during the COVID-19 epidemic.

\section{CLINICAL CHARACTERISTICS OF COVID-19 IN CHILDREN \\ Epidemiological characteristics ${ }^{912}$}

Patients with COVID-19 infection are the primary sources of infection, but the incubation period patients and patients with asymptomatic infection are also sources of infection. The transmission is mainly through the respiratory tract, by droplets when patients cough, speak loudly, or sneeze. The virus can also be transmitted through close contact (such as touching the mouth or nose, or conjunctiva by contaminated hands). Aerosol and fecal-oral transmission remain to be confirmed. In addition, it is currently uncertain whether the virus can be transmitted through mother-to-child vertical transmission 
or through breast milk. The population is generally susceptible. The elderly and those with basic diseases are prone to severe symptoms and complications when infected. Infants and young children are likely to have mild symptoms when infected.

\section{Clinical manifestations and subgroups ${ }^{912} 18$}

The incubation period is $1-14$ days, and most commonly 3-7 days. Fever and coughing are often observed, as well as fatigue, myalgia, stuffy nose, runny nose, sneezing, sore throat, headache, dizziness, nausea, vomiting, abdominal pain and diarrhea. Blood routine leukocyte counts and absolute lymphocyte counts are mostly normal. The lung image is characterized by ground glass shadow on the outside of the lung. Mild manifestations mainly show acute upper respiratory infections, including fever, fatigue, myalgia, cough, sore throat, runny nose, and sneezing. While physical examination reveals congestion in the pharynx, no positive signs exist in the lungs. Some children may have no fever or may have only digestive symptoms such as nausea, vomiting, abdominal pain, and diarrhea; the common cases show symptoms of pneumonia, frequent fever, and cough, and mostly dry cough followed by sputum cough. Some may have wheezing, but no obvious hypoxia such as shortness of breath. Lungs can hear sputum or dry snoring and/or wet snoring. Some children did not have any clinical symptoms and signs, but chest CT showed lung lesions, which were diagnosed subclinical. Most cases with children are mild or subclinical; severe cases show early respiratory symptoms such as fever and cough and can be accompanied by gastrointestinal symptoms such as diarrhea. This usually progresses around one week. Patients have difficulty breathing, have central cyanosis, or do not take oxygen hypoxic manifestations such as inferior pulse blood oxygen saturation $<0.92$; critically ill cases can progress rapidly to acute respiratory distress syndrome (ARDS) or to respiratory failure and also may develop shock, encephalopathy, myocardial injury or heart failure, coagulopathy, acute kidney injury, and other multiple organ dysfunctions, which can be life threatening. ${ }^{18}$

\section{Criteria for the diagnosis of suspected and confirmed cases in children ${ }^{12}$}

Suspected cases: with any one of the following epidemiological histories and any two of the clinical manifestations, diagnosis can be made. Epidemiological history: (1) traveling or living in areas with continuous local transmission cases within two weeks before the onset; (2) contact with patients in fever or with respiratory symptoms from areas with continuous local transmission cases within two weeks before the onset; (3) have a history of close contact with confirmed or suspected cases of COVID-19 within two weeks before the onset of disease; (4) have clustering onset: in addition to this child, there are other patients with fever or respiratory symptoms, including suspected or confirmed COVID-19 cases; (5) babies given birth by pregnant women with suspected or confirmed neonatal
COVID-19. Clinical manifestations: (1) fever, fatigue, dry cough, some children may have no fever or low fever; (2) above-mentioned imaging manifestations; (3) white blood cell count is normal or decreased in the early stage of onset, or the lymphocyte count is reduced.

Confirmed cases: suspected cases can be diagnosed if they meet any of the following pathogenic test results: (1) real-time fluorescence PCR detection of SARS-CoV-2 nucleic acid samples in throat swabs, sputum, stool, or blood; (2) above-mentioned sequencing and known virus genes SARS-CoV-2 are highly homologous; the above specimens were isolated and cultured to SARS-CoV-2 particles.

\section{Basic classification of pediatric surgery and principles of admission during the COVID-19 epidemic}

Emergency surgery is a surgery that should be performed immediately after examination and evaluation of lifethreatening diseases, such as severe trauma, acute abdominal disease, and testicular torsion. Even if a child has been diagnosed or suspected with COVID-19, the surgery should be performed in the designated hospital under the effective protection (see below). If pediatric surgeons are not available in the designated hospital, pediatric surgeons from other hospitals can conduct the operation after obtaining permission in the designated hospital.

Limited-term surgery, a surgery which should be conducted in a limited time, is carried out to treat severe or rapidly progressive diseases, including advanced cancers, biliary atresia, and giant hydronephrosis with developing kidney function continues declining. If a patient is diagnosed, or suspected with COVID-19, the operation can be postponed appropriately. The operation should be scheduled once the two results of nucleic acid tests are negative after two weeks or once the COVID-19 expert group has confirmed the exclusion or recovery. However, it is still recommended to perform the operation under essential protection (see below). If the suspected case is ruled out or if the local epidemic situation has been effectively controlled, it is recommended that these patients receive operations in batches under certain protection. Meanwhile, a prearranged planning should be prepared in the event that patients which received operations develop clinical symptoms and signs of COVID-19 postoperatively. Once these patients are confirmed or highly suspected by radiological examination and nucleic acid test, person in close contact with the patients should be effectively traced and isolated, and operation rooms should be disinfected. In that way, the risk of COVID-19 transmission can be highly minimized.

Elective surgery is performed for diseases that can be arranged after months or even longer without serious outcomes, such as incarcerated inguinal hernia or hypospadias. To avoid the risk of COVID-19 transmission, it is recommended that these surgeries should be postponed until the outbreak is under control. 
Outpatient management of pediatric surgery ${ }^{6} 15$

According to the epidemic characteristics of COVID-19 and the experience accumulated in clinical practice, some patients can be infected but not present with symptoms. A few parents may conceal the history of coming from an affected area or of contact with patients with COVID-19; therefore, the protective work of COVID-19 is still significant.

Outpatient personnel protection: outpatient personnel including patients and their dependents, clinical staff, cleaners and persons may in the outpatient hall. Pretreatment education should be strengthened by the official website, WeChat public account, outpatient hall and other channels; moreover, one dependent accompany policy is carried out these days. Patients with respiratory symptoms, fever, or the history of close contact with patients with COVID-19 within 14 days should be referred to the COVID-19 specialist clinic. Patients and dependents are advised to wear masks and observe the clinic order. Clinical staff and cleaners should carry out the following protective measures such as wearing work apparel, surgical masks or N95 masks, disposable round caps, and gloves. ${ }^{19}$

Outpatient equipment and environmental disinfection: ${ }^{20}$ there are few invasive examination equipment in a surgical outpatient clinic. However, the attention should be paid to the disinfection of the surface of relevant instruments and equipment, including tables and chairs, examination beds and other items. It is recommended to use sodium hypochlorite disinfectant to wipe the tables, chairs, and surface of the things used before and after the outpatient treatment. The purification of the air in the outpatient clinic is mainly based on ventilation. However, the natural ventilation of clinic is somewhere unavailable. Thus, mechanical ventilations are recommended in the units where conditions permit. Attention should be paid to the regular cleaning of ventilation equipment. After daily clinic work, ultraviolet irradiation or ultralow volume spraying should be used to thoroughly disinfect the clinic places. ${ }^{21} 22$

Distinguishing patients with COVID-19 before admission (including dependents): during the COVID-19 epidemic, expect the routine pediatric surgical information, we should also inquire about the patient's epidemiological history in detail, especially the contacting history of patients and dependents children with people from COVID-19 epidemic area and whether there are suspected or confirmed cases in their community, and so on. We should pay more attention to children with history of contact. Meanwhile, the presence of fever, dry cough, and dyspnea are observed. Blood test and chest imaging examination are listed as routine examinations. Any abnormality, the expert group of COVID-19 diagnosis, and treatment should be consulted immediately. No missing case of COVID-19 diagnosis is recommended as far as possible. Actually, some pediatric surgical diseases have similar clinical manifestations, especially the infectious diseases, outpatient physicians should check carefully. If patient performs suspicious manifestations, they should be transferred to a fever clinic in accordance with the protection principle. Once the suspected or confirmed diagnosis is made, the patient should be quarantined, treated, and reported immediately according to regulations.

\section{IN-PATIENT AREA PROTECTION 92122}

Admission of the emergency patients with confirmed or suspected infection of COVID-19

The technical guidelines for the prevention and control of COVID-19 infections in medical institutions (first edition) must be conducted strictly. ${ }^{17}$ So far, the isolation wards have already been set up in the designated hospitals according to the National standards requirements which including the building layout, routine procedures, and contacts, droplet, airborne isolation precautions, and so on. Critically ill children should be admitted in the isolated intensive care unit (ICU) or in the isolated wards equipped with monitoring and rescue facilities. Mixed treatment with other sick children suffered from non-COVID-19 is not allowed. Medical staffs should strictly obey the principles of personal protective equipment use. ${ }^{17}$ The children diagnosed or suspected with COVID-19 should be placed in the specific isolated wards, no matter what surgical specialties disease they have. Perioperative management should be completed jointly with various specialized surgeons and with physicians and nurses from the isolated wards. In this situation, it is recommended to choose shorter duration as a priority in the surgical approach to minimize contact with the patient and to reduce the risk of cross infection. For instance, if the surgeon considered that the duration of open surgery is shorter than that of laparoscopic surgery according to his own experience, open surgery should be chosen accordingly. In addition, open surgery requires simpler surgical instruments, shorter preparation time, and easier postoperative sterilization as compared with these for laparoscopic surgery.

\section{Admission of the patients preliminary excluded the possibility} of COVID-19 infection

An examination of the National and Provincial Health Commission official websites shows that there are no guideline documents or recommendations for the patients receiving limited-term surgery with initially exclude of COVID-19 infection or precautions to prevent transmission of infectious agents. Thus, the proposal of the article mainly refers to "Guideline for infection control for H1N1 influenza in health care."23

Ward protection: (1) partition isolation: to avoid cross infection of hospitalizing children or their accompanying parents in COVID-19 incubation period or with asymptomatic infection within wards, it is recommended that all the rooms in the ward should be set as a single bed during the epidemic period. If the conditions are limited, $>1 \mathrm{~m}$ spatial separation between beds is advised in 
shared rooms according to the precaution management. (2) Proper air and floor disinfection: natural ventilation by opening windows twice a day, at least one hour each time in the rooms where patients were placed; ultraviolet disinfection once or twice a day, at least 30 min each time in empty room. Doors and windows are closed during the disinfection and after disinfection, and windows are opened for ventilation. For windowless rooms or storage rooms, regular mechanical exhaust and ultraviolet disinfection are required. The ventilation should be ensured in recovery room for 24 hours by exhaust equipment. The frequency of ultraviolet disinfection changes from once a day to twice a day for dressing rooms, treatment rooms and recovery rooms. Disinfectant with effective chlorine in concentration of $1000 \mathrm{mg} / \mathrm{L}$ can be wiped or sprayed twice a day on the floor and in the corridors of the ward. Wet cleaning shall be kept during ground wiping, and disinfection shall be carried out promptly in case of pollution. (3) Proper cleaning and disinfection of surfaces in patient-care areas: the surfaces frequently touched by children and their parents in the ward (such as bedrails, headboard, bedside tables, pagers, monitors, doorknobs, and so on) should be disinfected with effective chlorine containing disinfectant using a concentration of $1000 \mathrm{mg} / \mathrm{L}$ for $30 \mathrm{~min}$ every day and then wiped by clean water. Corrosion-susceptible items are sterilized by wiping with $75 \%$ ethanol twice. Thermometers, stethoscopes, sphygmomanometers, and other medical equipment (such as goggles without obvious pollution) should be disinfected with $75 \%$ ethanol twice after use. (4) Medical staff precautions: medical staffs are at risk of cross infection as they need to face many people, including the patients, their parents, and other medical staff. All departments of a healthcare should educate and train healthcare personnel on the principles and recommended practices of COVID-19 Standard Precautions. Reasonable shift arrangement and shift system implementation are also required. Medical staffs should self-monitor whether they have fever, dry cough, fatigue, and other symptoms and should report their status to the relevant departments of healthcare daily. If any associated symptoms are present, essential monitor and isolation procedures should be performed as soon as possible. Healthcare personnel should wear appropriate personal protective equipment, such as surgical mask, goggles, face shield, disposable caps, gloves, before carry out routine medical work. Precautions should be strictly observed according to "The technical guidelines for the prevention and control of the new coronary virus infections in medical institutions (first edition)" when healthcare workers perform the procedures such as change dressing, remove drainage or change ostomy bag and expose to patients' blood, body fluids or excretions. Proper hand washing is effective precaution in preventing transmission of viruses. Healthcare personnel should abide by the principles of hand hygiene before and after having direct contact with patients and after removing gloves. ${ }^{24}$ (5) Precautions for non-healthcare professionals. Gatherings of many non-healthcare professionals increase the transmission risk of infectious agents in surgical wards. Thus, it is crucial to strengthen the precautions for patients, their parents, accompanying persons, and the cleaning staff. Health education for hospitalized children and their parents should be strengthened in each department, and patients' parents need to sign the "Commitment for the special epidemic notification" before admission. Only one parent was recommended to accompany the children, and any visitor should be refused. Patients and accompanying family members should reduce their activity space to lessen the transmission risk. Wearing a mask is necessary in the ward public area. When coughing or sneezing, one's month and nose need to be covered with a tissue or with one's elbow. Washing hands and disinfection is required after contact with respiratory secretions, before meals, and after toileting. Keep the room ventilated. The temperature of the patients, their parents, and the cleaning staff need to be measured twice a day, and any associated symptom should be reported to the special medical professional, such as fever, cough, or fatigue.

\section{PERIOPERATIVE PROTECTION ${ }^{6}$}

\section{Protection for confirmed or suspected children who need} emergency surgery

Preoperative preparation: wearing warning signs and surgical masks is required for patients and their accompanying parents consistent with hospital uniform management rules; medical records should be marked with warning labels. A negative pressure transfer vehicle should be used to deliver the patients with responsible physician wearing protection gear to the special isolation zone, which is set up by operating room, through the special passageway and elevator to avoid contamination of the operating room. (1) Operating room preparations: the operation should be carried out by less participants in the designated negative pressure or special operating room designed for infection with conspicuous signs posting at the door. And the entry and exit for noninvolvement personnel should be declined. The operating room is equipped with a thermometer to monitor the body temperature of each before the beginning of operation. The negative pressure should be kept at -5 $\mathrm{Pa}$. If negative pressure operating room is not available, the surgery should be performed in the operating room which is nearest to the special channel and the most marginal area with turning off the laminar flow. (2) Participant preparations: training for pre-employment and the use of personal protection gear is required for participants, including surgeons, anesthesiologists, and nurses, who are in charge of filling Surgical Participation Registration Form and record work. According to the three-level protection rules, health workers and anesthesiologists are required to wear a double-layer disposable cap, medical mask, disposable isolation operating coat and medical protective suits, goggles, or masks, double-layer sterile gloves, and over-the-knee gloves. It is 
recommended to wear protective suits under supervision of special personnel according to the "Health Workers Wear Protection Gear Procedures." (3) Surgical and anesthetic equipment preparation: before operation, full communication should be carried out and items should be prepared enough to avoid the flow of personnel and articles as much as possible, which affects the operating room negative pressure efficiency. Disposable diagnosis and treatment supplies, medical appliances and nursing supplies are preferred. Surgical and anesthetic instruments and equipment should be prepared as required as specialty by surgery. It is crucial to use all medical supplies according to the principle of dedicated personnel.

Intraoperative management: (1) anesthetic management and protection: the situation of children and the operation requirements need to be considered when choosing the appropriate anesthetic method. It is recommended to choose anesthetic methods and medicine with minimal impact on child on the basis of ensuring safety and painlessness of children, as well as safety of medical workers meanwhile. For pediatric patients that cry more often and do not cooperate, appropriate sedation can be applied before surgery to reduce the risk of transmission of saliva and droplet. The three-level protection measures are required for anesthesiologists, and one more layer of gloves is necessary before intubation during the endotracheal intubation for general anesthesia, then be removed after the completion of intubation. Medication should be used on those who have venous access as soon as possible to get sedation after entering the room, and sevoflurane inhalation can be applied on those without venous access to get sedation, followed by the establishment of venous access. Before anesthesia induction, double layer of warm and moist gauzes should be used to cover the mouth and nose of patient, followed by mask sustained high flow preoxygenation. It is recommended that to choose rapid anesthesia induction, moderate sedation and sufficient muscle relaxant are necessary to avoid choking cough, and intubation should be performed by skilled pediatric anesthesiologists under optimal conditions for high success rate. In case of difficult airway, the laryngeal mask should be placed after the failure of the first endotracheal intubation (attempt) to avoid the risk of infection caused by repeated attempts of endotracheal intubation. To avoid being close to operating area, the use of visual laryngoscope will be required. The patients with oral secretions could be cleaned with a closed suction system after completion of endotracheal intubation to avoid secretion contamination, if there is no obstruction of respiratory tract. Monitoring should be strengthened during operation, and severe patients are required to be closely monitored and dealt with in time due to the possible existence of acute lung injury, ARDS, heart failure, acid-base imbalance, and electrolyte disorders. At the end of the operation, the endotracheal tube should be removed under deep sedation with strengthening monitoring. It is recommended that complete the sputum suction work before the child wake up and appropriate injection of lidocaine before extubation. The mouth and nose of patient should be covered with two warm and moist gauzes to reduce the secretion spatter caused by choking cough as much as possible. Antagonists are not recommended. The filter at the end of the tracheal catheter is required to be kept during extubation. The work of postoperative analgesia is necessary to reduce crying and restlessness of children after extubation. The patient needs to be transferred to the isolation ward directly for continued treatment when Steward recovery scores are $\geq 5$ points after extubation with an observation in the operation. Those severe patients need to be transferred to isolation ICU directly with keeping trachea catheter. It is recommended to deepen the anesthesia or add appropriate dosage of muscle relaxant before transferring. (2) Intraoperative precautions: general open operations refer to the "Health workers Wear Protection Gear Procedures." The contamination, caused by mixture of gas when cutting tissues, should be avoided during laparoscopic surgery in operating room. In addition to the precautions of patients' blood, secretions, and excrements during the operation, special attention should be paid to the aerosol produced when using electrosurgical equipment such as electric knife. The electric knife should be used matching with the minimum effective power and the smoke absorbing device. Doctors and nurses should operate accurately and normatively to avoid injuries caused by knife, suture needle, and other instruments. Participants are asked to remove the protective equipment according to the "Health workers Wear Protection Gear Procedures" after the operation, and leave through the special passageway.

Postoperative protection: (1) transshipment management: patients should be transferred postoperatively to the designated isolation ward for treatment through the prescribed route after report to the hospital infection management department and medical affairs department. According to the Double-medical team leader system, the patient who was transferred to the isolation ward should be managed by leaders from isolation ward and surgical team. Precautions should be strictly observed during transshipment. Transfer flatbed covered with disposable sheets is required to be thoroughly disinfected by disinfectant with effective chlorine in concentration of $2000 \mathrm{mg} / \mathrm{L}$ after transfer. (2) Operating room management: the operation room is fumigated and disinfected with peroxyacetic acid after turning off the operation laminar flow and air supply. The high efficiency filter of the operation is required to be replaced in time by notifying laminar flow engineers and technicians. Contaminants should be removed from floor, wall, and articles in the operating room, which should be sprayed and wiped by disinfectant with effective chlorine in concentration of $2000 \mathrm{mg} / \mathrm{L}$, at least $30 \mathrm{~min}$ each time. Then, the floor should be mopped by clean water. The surface and air sampling test in operation should be carried out after disinfection by contacting the infection management department, and operation can be used again after the 
results are qualified. Turn on laminar flow and ventilation for at least 2 hours. (3) Management of objects and specimens during operation: after operation, the reusable devices and cloths need to be sprayed with moisturizer, sealed, and packed with double-layer of yellow plastic bag, affixed with the "SARS-CoV2" logo and the name of the article, and placed separately. The supply center staffs are called to collect them in time for the subsequent disinfection. Disposable articles, medical protection equipment, and body fluid, flushing fluid generated by suspected or confirmed patients with SARS-CoV2 infection should be placed and sealed in double-layer of yellow medical waste bags, affixing with the "SARS-CoV2" logo outside. Then, those bags should be placed separately and processed as infectious medical waste. The specimen bag, labeling with "SARS-CoV2," was sent to the pathology department for diagnosis. (4) Medical record of operation: in addition to the information of patients, the "Infectious Diseases Registration Form" should be filled by surgeon in time, as well as updating of the diagnosis of suspected cases and the surgical recovery status of patients. (5) Follow-up management: patients are transferred to the designated negative pressure isolation ward. Precautions should be strictly observed according to "Technical Guidelines for the Prevention and Control of New Coronavirus Infections in Medical Institutions (first edition)" when medical workers entering the isolation ward for rounds, dress changing, and nursing. ${ }^{17}$ (6) Discharge criteria: different from the usual surgical discharge criteria, the consultation of hospital COVID-19 Expert Group needs to be considered that whether can be discharged or need continuous home isolation with disease control and monitoring for those patients who still need further isolation after surgery according to the regulations.

Protection for the patients who need limited-time surgery and preliminary excluded possibility of suspected or confirmed with COVID-19 infection

During the COVID-19 epidemic period, a higher level of protection is needed to avoid large-scale infections among medical staff due to missed cases. In addition to the routine operations as usual, additional protective standards are required during the perioperative period, including: ${ }^{23}$ (1) isolation of all patients' blood, body fluids, secretions, and excreta is necessary because of their infectivity. Precautions must be performed when exposed to the above-mentioned substances or to nonintact skin and mucous membranes. (2) Emphasis on two-way precaution. It is necessary to prevent the transmission of disease from patients to medical staff and also to prevent the reverse transmission from medical staff to patients.

Specific measures of standard protection ${ }^{23}$ include: (1) hands cleaning and disinfection, which should meet the requirements of the "Hand Hygiene Specification for Health Personnel," ${ }^{24}$ is an important measure to prevent contact transmission. (2) Wearing gloves is required when exposed to blood, body fluids, secretions, excreta, and other substances, as well as articles contaminated by them. (3) Wash hands immediately after removing the gloves. (4) Wearing surgical masks, protective glasses or masks, and isolation gown or waterproof aprons is necessary to protect work clothes, face, and eyes of medical workers from contaminating by spatter of blood, body fluids, secretions, and so on. (5) Special attention should be paid to all sharpeners to prevent stab wounds. (6) Correct disinfection and sterilization measures should be taken for the medical devices and appliances after use. (7) During the epidemic, it is recommended to wear medical protective masks, as well as protective glasses or masks, gloves, isolation gown, protective suits, shoe gloves, and so on, especially when performing endotracheal intubation for general anesthesia. Protection gear should be used correctly in accordance with the requirements of the "Technical Specification for the Isolation of Medical Institutions." ${ }^{25}$

Management of patient who had postoperatively clinical symptoms and suspected or confirmed of COVID-19 infection: (1) the above patients, who have even nucleic acid tested negative for the virus once, should be reported to the relevant departments of hospital immediately. (2) The patients should be transferred to the isolation ward as soon as possible. (3) The related ward, operating room, and surgical instruments need to be disinfected as required, and treatment of other patients is not allowed in the above-mentioned area. (4) All medical workers and non-medical workers in the ward with a history of contact should be retrospectively and quarantined for strict observation for 14 days.

\section{Discharge precautions}

The condition of child with pediatric surgical diseases changes rapidly. Patients will be asked for further consultation within 1-2 weeks after the surgery. However, during the epidemic, convenient contact medias, such as WeChat, the official website, and department phone numbers, can be provided for parents to consult for the recovery status of the patients. Outpatient consultation can be postponed until the epidemic is under control, and traffic isolation is completely removed if no urgent treatment is needed.

\section{General elective surgery, day surgery, and outpatient clinic surgery}

These operations need to be delayed until the notification from the superior department of government; however, online or remote appointments can be registered in various ways to carry out preoperative health education. When an emergency occurs, such as hernia incarceration, patients should go to the hospital immediately. After receiving the notification from the superior department, it is recommended to make appointments at different times and to perform the operations gradually to avoid crowd gathering in a short time in the hospital.

\section{CONCLUSION}

Under the current epidemic situation, patients of pediatric surgery need special protection. For patients with 
confirmed or suspected SARS-CoV infection, limitedterm surgery should be postponed. If emergency surgery is necessary, protection should be carried out strictly following the strategies described in this article. Elective surgery will be resumed after the epidemic situation is gradually under controlled.

Contributors DT, JT, JW, CQ and WW proposed the work and wrote the strategy formulation of the article. JH, HZ, JW, ZX, DZ and JF wrote the rest of the article. QS is the guarantor of the article.

Funding This article was supported by Zhejiang University special scientific research fund for COVID-19 prevention and control.

Competing interests None declared.

Patient consent for publication Not required.

Ethics approval Not required for this review article.

Provenance and peer review Not commissioned; externally peer reviewed.

Data availability statement Data sharing not applicable as no datasets generated and/or analyzed for this study.

Open access This is an open access article distributed in accordance with the Creative Commons Attribution Non Commercial (CC BY-NC 4.0) license, which permits others to distribute, remix, adapt, build upon this work non-commercially, and license their derivative works on different terms, provided the original work is properly cited, appropriate credit is given, any changes made indicated, and the use is non-commercial. See: http://creativecommons.org/licenses/by-nc/4.0/.

ORCID iD

Zheming Xu http://orcid.org/0000-0003-4284-3594

\section{REFERENCES}

1 Gorbalenya AE, Baker SC, Baric RS, et al. Severe acute respiratory syndrome-related coronavirus: the species and its viruses- a statement of the coronavirus Study Group. bioRxiv 2020.

2 National Health Commission of the People's Republic of China, National Administration of Traditional Chinese Medicine. Pneumonia diagnosis and treatment plan for new coronavirus infection (trial version 5 revision) (in Chinese). Available: http://www.nhc.gov.cn/ yzygj/s7653p/202002/d4b895337e19445f8d728fcaf1e3e13a/files/ ab6bec7f93e64e7f998d802991203cd6.pdf [Accessed 12 Mar 2020].

3 National Health Commission of the People's Republic of China. Announcement of National Health Commission of the People's Republic of China; No.1, 2020 (in Chinese). Available: http:// www.nhc.gov.cn/jkj/s7916/202001/44a3b8245e8049d2837a4f27 529cd386.shtml [Accessed 12 Mar 2020].

4 Guo WY, Yuan JP, Fu T, et al. Perioperative prevention and control strategies for surgical patients in the context of new coronavirus pneumonia (in Chinese). Chin J Dig Surg 2020;19:E001.

5 Li Y, ZF L, Mao QX, et al. Consensus on emergency surgery and infection prevention and control for severe trauma patients with 2019 novel coronavirus pneumonia. Chin J Trauma 2020;36:1-7. (in Chinese).

6 Tao KX, Zhang BX, Zhang P, et al. [Recommendations for general surgery clinical practice in novel coronavirus pneumonia situation]. Zhonghua Wai Ke Za Zhi 2020;58:E001. (in Chinese).

7 Cai JH, Wang XS, YL G, et al. First case of 2019 novel coronavirus infection in children in Shanghai (in Chinese). Chin J Pediatr 2020;58:E002.

8 Deng HL, Zhang FY, Wang Y, et al. Novel coronavirus infection in 2 children with cases (in Chinese). Chin Pediatr Emerg Med 2020;23:E004.
9 The Society of Pediatrics, Chinese Medical Association. The editorial board, Chinese Journal of pediatrics. recommendations for the diagnosis, prevention and control of the 2019 novel coronavirus infection in children (first interim edition). Chin J Pediatr 2020;58:E004. (in Chinese).

10 Bai Y, LP Y, Qing Q, et al. 20 questions and answers for prevention of severe acute respiratory syndrome coronavirus infection in children (in Chinese). Chin J Appl Clin Pediatr 2020;35:86-91.

11 Zeng M, Zhai XW, Wang JS, et al. Novel coronavirus infection: pediatric professionals' perspectives and action. Chin $\mathrm{J}$ Infect Dis 2019;2020:E003. (in Chinese).

12 Chen Z, Fu J, Shu Q, et al. [Diagnosis and treatment recommendation for pediatric coronavirus disease-19]. Zhejiang Da Xue Xue Bao Yi Xue Ban 2020;49:0. (in Chinese).

13 Fang F, Luo XP. Facing the pandemic of 2019 novel coronavirus infections: the pediatric perspectives. Chin J Pediatr 2020;58:81-5. (in Chinese).

14 Jiang Y, BP X, Jin RM, et al. Diagnosis, treatment and prevention of 2019 novel coronavirus infection in children: experts' consensus statement (first edition). Chin J App/ Clin Pediatr 2020;35:81-5. (in Chinese).

$15 \mathrm{HZ}$ X, Chen SH, JF F, et al. Advices on the prevention and control of nosocomial infection of novel coronavirus within children's hospitals. Chin J Hosp Admin 2020;36:E001. (in Chinese).

16 Shen K, Yang Y, Wang T, et al. Diagnosis, treatment, and prevention of 2019 novel coronavirus infection in children: experts' consensus statement. World J Pediatr 2020;22.

17 National Health Commission of the People's Republic of China. Announcement on the issuance of technical guidelines for the prevention and control of the new coronary virus infections in medical institutions (first edition) (in Chinese). Available: http:// www.nhc.gov.cn/xcs/yqfkdt/202001/b91fdab7c304431eb082d678 47d27e14.shtml [Accessed 12 Mar 2020].

18 Chen Z-M, Fu J-F, Shu Q. New coronavirus: new challenges for pediatricians. World J Pediatr 2020. doi:10.1007/s12519-020-003464

19 National Health Commission of the People's Republic of China. The new coronary virus transmission pathways and the Guideline for prevention (in Chinese). Available: http://www.nhc.gov.cn/jkj/s3578/ 202001/9e73060017d744aeafff8834fc0389f4.shtml [Accessed 12 Mar 2020].

20 National Health Commission of the People's Republic of China, National Administration of the People's Republic of China. Pneumonia diagnosis and treatment plan for new coronavirus infection (trial version 5 revision) (in Chinese). Available: http://www. gov.cn/zhengce/zhengceku/2020-02/05/content_5474791.htm [Accessed 12 Mar 2020].

21 National Health Commission of the People's Republic of China Standard for air cleaning techniques in health care (in Chinese). Available: http://www.nhc.gov.cn/wjw/s9496/201204/54511/files/ 8df30d0236d3421c87492786c55c26e7.pdf [Accessed 12 Mar 2020].

22 National Health Commission of the People's Republic of China. Regulation for prevention and control of healthcare associated infection of airborne transmission disease in healthcare facilities (in Chinese). Available: http://www.nhc.gov.cn/ewebeditor/uploadfile/ 2017/01/20170119150530360.pdf [Accessed 12 Mar 2020].

23 National Health Commission of the People's Republic of China. Guideline for infection control for H1N1 influenza in health care (in Chinese). Available: http://www.nhc.gov.cn/yzygj/s3585/200905/83f9 d87498bd4dbca0bebb6301be4c1e.shtml [Accessed 12 Mar 2020].

24 National Health Commission of the People's Republic of China. Standard for hand hygiene for healthcare workers in healthcare settings (in Chinese). Available: http://www.nhc.gov.cn/ cmsresources/mohyzs/cmsrsdocument/doc5839.pdf [Accessed 12 Mar 2020].

25 National Health Commission of the People's Republic of China. Technique standard for isolation in hospital (in Chinese). Available: http://www.nhc.gov.cn/cmsresources/mohyzs/cmsrsdocument/ doc5841.pdf [Accessed 12 Mar 2020]. 